\title{
Multiscale modeling of nano/micro systems by a multiscale continuum field theory
}

\author{
Xiaowei Zeng • Xianqiao Wang • James D. Lee • \\ Yajie Lei
}

Received: 8 March 2010 / Accepted: 29 August 2010 / Published online: 16 September 2010

(C) The Author(s) 2010. This article is published with open access at Springerlink.com

\begin{abstract}
This paper presents a multiscale continuum field theory and its application in modeling and simulation of nano/micro systems. The theoretical construction of the continuum field theory will be briefly introduced. In the simulation model, a single crystal can be discretized into finite element mesh as in a continuous medium. However, each node is a representative unit cell, which contains a specified number of discrete and distinctive atoms. Governing differential equations for each atom in all nodes are obtained. Material behaviors of a given system subject to the combination of mechanical loadings and temperature field can be obtained through numerical simulations. In this work, the nanoscale size effect in single crystal bcc iron is studied, the phenomenon of wave propagation is simulated and wave speed is obtained. Also, dynamic crack propagation in a multiscale model is simulated to demonstrate the advantage and applicability of this multiscale continuum field theory.
\end{abstract}

Keywords Atomistic simulation .

Continuum field theory $\cdot$ Finite elements .

Numerical simulation $\cdot$ Mechanical behavior .

Multiscale simulation

\section{Introduction}

According to Stan and Yip [1], from the computational point of view, the grand challenge of material simulations is to

\section{Zeng (凶)}

Department of Civil and Environmental Engineering,

University of California at Berkeley, Berkeley, CA 94720, USA

e-mail: xwzeng@berkeley.edu

X. Wang · J. D. Lee · Y. Lei

Department of Mechanical and Aerospace Engineering,

The George Washington University, Washington, DC 20052, USA understand, predict, and control one mole of substance $\left(6.02 \times 10^{23}\right.$ atoms or molecules $)$, for one second, by the year 2015. Moreover, at atomistic level, the time scale is femtosecond, it will require $10^{15}$ time steps to reach one second. Both atomistic simulations and classical continuum theories have their limitations, i.e., atomistic simulations can be accurate, but are inefficient and impractical for large systems and long timescales even with latest computing power; similarly continuum theories are efficient for large systems, however, they are inaccurate for miniaturized devices with atomistic features, major challenges exist for simulating micro/nano-scale systems over a realistic range of time, length, and temperature. The development of accurate and efficient computational design tools that can bridge various lengths and time scales are central for further advances in micro/nano-scale systems.

A number of multiscale simulation methods have been developed in the past decade. The quasi-continuum (QC) method, proposed by Tadmor et al. [2], is remarkably successful in many applications. The QC method is based on standard finite elements and constitutive equations derived from atomistic interactions and it seamlessly couples the atomistic and continuum realms. There are two versions of quasi-continuum methods: a local version of QC method applicable at mesoscale and a nonlocal version of QC method that is designated for atomistic scale simulation. The local version of QC method is basically a straightforward application of the Cauchy-Born rule in linear finite element method, and the use of Cauchy-Born rule to extrapolate material constitutive relations may be dated back in 1980s such as Ericksen [3]. A main challenge of the QC method is how to couple the macroscale local QC method with the microscale non-local QC method. The QC potential energy leads to some non-physical effects in the transition region. Specifically, taking derivatives of the energy functional to obtain 
forces on atoms and FE nodes leads to so-called ghost forces in the transition region. The origin of these ghost forces lies precisely in the assumption of locality in the continuum region and the local/non-local mismatch in the transition region.

By recognizing that FEM based continuum coarse grain models may have difficulties to achieve smooth transition from atomistic description to continuum description when temperature effects are important, Rudd and Broughton $[4,5]$ proposed a discrete coarse grain model- the so-called coarse-grained molecular dynamics (CGMD), in which the degrees of freedom reduction is developed as a more natural extension of the underlying discrete molecular dynamics. The CGMD approach is based on a statistical coarse-graining prescription. Their coarse-grain formulation employs a finite element representation, and the dimension reduction is done in the partition function by integrating out the excess atomic degrees of freedom. The CGMD approach produces equations of motion for the nodal fields are not derived from the continuum model but from the underlying atomistic model. The nodal fields represent the average properties of the corresponding atoms, and equations of motion are constructed to describe the mean behavior of underlying atoms that have been integrated out. One important underlying principle of CGMD is that it assumes that the system is under thermodynamic equilibrium, and the classical equilibrium ensemble condition is imposed to obey the constraint that the position and momenta of atoms are consistent with the mean displacement and momentum fields. Although both CDMD and QC are developed to couple FE and atomistic models, the QC method is mainly applicable to zero-temperature calculations, whereas the CGMD is designed for finite-temperature dynamics. The QC model has shown its success in many applications, but the CGMD approach has yet to show its wider applicability and versatility.

The first concurrent multiscale simulation method was probably the method of Macroscopic Atomistic Ab initio Dynamics (MAAD) proposed and developed by Abraham, Broughton, Bernstein, and Kaxiras [6-8], and they successfully used MAAD method to simulate crack propagation in Silicon. This multiscale methodology dynamically couples different length scales which ranges from the atomic scale region at the crack tip, treated with a quantum-mechanical tight-binding (TB) approximation method to model bond breaking; through the microscale region near the crack tip, treated via the classical molecular dynamics (MD) method; and finally to the mesoscale/macroscale region away from the crack tip, treated via the finite element (FE) method in the context of continuum elasticity. There are two more handshaking regions (FE/MD, MD/TB) exist in the interfaces. After the total Hamiltonian of the system being computed, equations of motion can be derived. A general problem associated with MAAD method is the spurious reflection of elas- tic waves (phonons) at the domain boundaries because MD region will generate phonons which are not represented in the continuum region and hence might be reflected at the FE/MD interface.

$\mathrm{Li}$ and $\mathrm{E}$ [9] proposed a multiscale methodology that can handle both dynamics and finite temperature effects, which is based on the framework of the heterogeneous multiscale method (HMM) developed by E and Engquist [10]. There are two major components in HMM: (2) the selection of a macroscale or mean field variables and their governing equations, and (2) the estimation or extrapolation of the values of the macroscale variables from homogenization or statistical averaging of microscale variables. In general the governing equations of the macroscale field variables should be chosen to maximize the efficiency in resolving the macroscale behavior of the system and minimize the complexity of coupling with the microscale model.

The bridging-scale method was proposed by Liu and coworkers [11-15], in which FE approximation co-exists with atomistic description. By employing the harmonic approximation, Liu and his colleagues have derived a multiscale boundary condition analytically or semi-analytically so that they have exact matching impedance at the multiscale interface, which will eliminate the spurious phonon reflections. Moreover, the multiscale boundary conditions are derived based on Langevin equation, so they can also take into consideration of thermal fluctuations. Xiao and Belytschko [16] have developed a bridging domain approach in which the compatibility between FE and MD are enforced based on constraints. In BSM method, the macroscale boundary condition at the fine/coarse grain interface is matched with that of the atomistic region through the lattice impedance techniques. The BSM method is exact when MD region is using linear atomic potentials and the constitutive relations in the macroscale are also linear. The multiscale boundary conditions can be employed within concurrent coupling methods to represent atomistic behavior in the continuum domain. This strategy can produce a smooth FE/MD coupling, without involving an artificial handshaking region at the atomistic/ continuum interface and a dense FE mesh scaled down the chemical bond lengths. Further development in the field of molecules dynamics and multiscale continuum theory can be found in references [17-20].

A main difficulty in the concurrent multiscale simulation is the spurious reflection of elastic waves due to the change of physical modeling as well as spatial resolution. $\mathrm{To}$ and $\mathrm{Li}$ [21] and $\mathrm{Li}$ et al. [22] have proposed a so-called perfectly-matched multiscale simulation(PMMS) to match the fine scale computation with the coarse scale computation. This is achieved by creating a MD-PML(perfectly-matched layer) region that can absorb any waves leaving the pure $\mathrm{MD}$ region so they will not introduce any artificial reflections. $\mathrm{Li}$ and his co-workers have applied the proposed PMMS method 


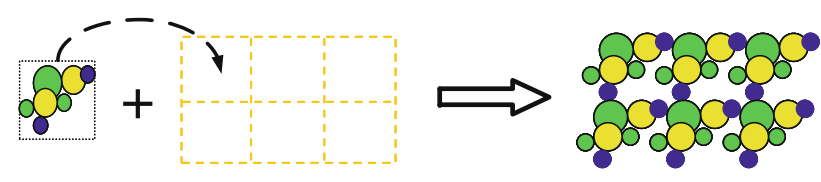

Fig. 1 Atomistic view of crystal structure

to simulate a screw dislocation passing through regions with different scales

A multiscale continuum field theory has been developed and documented over a series of publications for concurrent atomistic-continuum modeling of material systems [23-31]. In the theory, atomistic definitions and continuous local density functions of fundamental physical quantities are derived. By decomposing atomic motion/deformation into homogeneous lattice motion/deformation and inhomogeneous internal atomic motion/deformation, and also by decomposing momentum flux and heat flux into homogeneous and inhomogeneous parts, field description of conservation laws at atomic scale has been formulated. Since the conservation equations obtained by Chen et al. [23,25] are valid at atomic scale, the field theory can reproduce time-interval averaged atomic trajectories and can be used to investigate phenomena and properties that originated at atomic scale. On the other hand, it is a continuum field theory in terms of time-interval averaged quantities; it can be applied to simulate phenomena at larger length and time scales.

The paper is organized in six sections: in Sect. 2 we shall introduce the multiscale continuum field theory; Sect. 3 is focused on the FEM implementation of the multiscale field theory, in Sect. 4 we shall discuss the simulation methodology; and in Sect. 5, a few numerical simulations are presented, and finally we shall conclude the current study in Sect. 6.

\section{A multiscale continuum field theory}

Crystalline solids are distinguished from other states of matter by a periodic arrangement of the atoms; such a structure is called a crystal lattice. The atomic view of a crystal is as a periodic arrangement of local atomic bonding units. Each lattice point defines the location of the center of a unit. The space lattice is macroscopically homogeneous. Embedded in each lattice point is a group of bonded atoms, the smallest structural unit of the crystal, cf. Fig.1. The structure of the unit together with the network of lattice points determines the crystal structure and hence the physical properties of the material.

Macroscopic quantities are generally described by continuous (or piecewise-continuous) functions of physical space coordinates $\mathbf{x}$ and time $t$. They are fields in physical space- time. Microscopic dynamic quantities, on the other hand, are functions of phase-space coordinates $(\boldsymbol{r}, \boldsymbol{p})$, i.e., the positions and momenta of atoms. For multi-element systems, there is more than one atom in a unit cell. Thus, one has

$$
\begin{gathered}
\boldsymbol{r}=\left\{\boldsymbol{R}^{k \alpha}=\boldsymbol{R}^{k}+\Delta \boldsymbol{r}^{k \alpha} \mid k=1,2,3, \ldots n,\right. \\
\alpha=1,2,3, \ldots v\} \\
\boldsymbol{p}=\left\{m^{\alpha} \boldsymbol{V}^{k \alpha}=m^{\alpha} \boldsymbol{V}^{k}+m^{\alpha} \Delta \boldsymbol{v}^{k \alpha} \mid k=1,2,3 \ldots n,\right. \\
\alpha=1,2,3, \ldots v\},
\end{gathered}
$$

where the superscript $k \alpha$ refers to the $\alpha$ th atom in the $k$ th unit cell; $n$ is the total number of unit cells in the system and $v$ is the number of atoms in a unit cell; $m^{\alpha}$ is the mass of the $\alpha$ th atom; $\boldsymbol{R}^{k \alpha}$ and $\boldsymbol{V}^{k \alpha}$ are the position and velocity vector of the $k \alpha$ atom, respectively; $\boldsymbol{R}^{k}$ and $\boldsymbol{V}^{k}$ are the position and velocity of the mass center of the $k$ th unit cell, respectively; $\Delta \boldsymbol{r}^{k \alpha}$ and $\Delta \boldsymbol{v}^{k \alpha}$ are the atomic position and velocity of the $\alpha$ th atom relative to the mass center of the $k$ th unit cell, respectively. The local density of any measurable phase-space function $\boldsymbol{a}(\boldsymbol{r}, \boldsymbol{p})$ can generally be defined as

$$
\begin{aligned}
\boldsymbol{A}\left(\boldsymbol{x}, \boldsymbol{y}^{\alpha}, t\right)= & \sum_{k=1}^{n} \sum_{\beta=1}^{v} \boldsymbol{a}\{\boldsymbol{r}(t), \boldsymbol{p}(t)\} \\
& \times \delta\left(\boldsymbol{R}^{k}-\boldsymbol{x}\right) \tilde{\delta}\left(\Delta \boldsymbol{r}^{k \beta}-\boldsymbol{y}^{\alpha}\right) \equiv \boldsymbol{A}^{\alpha}(\boldsymbol{x}, t) .
\end{aligned}
$$

The first delta function in Eq. (2) is a localization function that provides the link between phase space and physical space descriptions. It can be a Dirac $\delta$-function by Irvine and Kirkwood [32], or a distribution function by Hardy [33], such as

$\delta\left(\boldsymbol{R}^{k}-\boldsymbol{x}\right)=\pi^{-3 / 2} l^{-3} e^{-\left|\boldsymbol{R}^{k}-\boldsymbol{x}\right| / l^{2}}$.

The field descriptions of the conservation equations and the constitutive relations (the interrelations between field quantities) are found to be independent of the choices of the localization function $[23,25,33]$. The second delta function in Eq. (2) is a Kronecker delta, which identifies $\boldsymbol{y}^{\alpha}$ to $\Delta \boldsymbol{r}^{k \alpha}$. It can be easily proven that the following normalization condition holds

$$
\begin{aligned}
& \int \delta\left(\boldsymbol{R}^{k}-\boldsymbol{x}\right) \tilde{\delta}\left(\Delta \boldsymbol{r}^{k \alpha}-\boldsymbol{y}^{\alpha}\right) d^{3} \boldsymbol{x}=1 \\
& \quad(k=1,2,3, \ldots n)(\alpha=1,2, \ldots v) .
\end{aligned}
$$

Also, it is obvious that the distribution function, Eq. (3), satisfies the following identity as the Dirac $\delta$-function does

$$
\frac{\partial \delta\left(\boldsymbol{R}^{k}-\boldsymbol{x}\right)}{\partial \boldsymbol{R}^{k}}=-\frac{\partial \delta\left(\boldsymbol{R}^{k}-\boldsymbol{x}\right)}{\partial \boldsymbol{x}} .
$$


Physical properties, such as thermodynamic properties and transport properties, refer only to average behavior. In the past, this was achieved through the theory of statistical mechanics. Averages were constructed by including many similar systems with different initial conditions. By this procedure, starting the system in any initial configuration would yield the same average quantities: this explains the reproducibility of experiments. Therefore, in statistical mechanics, a macroscopic field quantity is defined as the ensemble average of an instantaneous dynamical function. Most current MD applications involve systems which are either in equilibrium or in some time-independent stationary state; where individual results are subjected to fluctuation; it is the well-defined averages over sufficiently long time intervals that are of interest. To smooth out the results and to obtain results close to experiments, measurements of physical quantities are necessary to be collected and averaged over finite time duration. Therefore, in deriving the field description of atomic quantities and balance equations, it is the time-interval averaged quantities that are involved. The time-interval averaged (at time $t$ in the interval $[t, t+\Delta t])$ local density function is defined as

$$
\begin{aligned}
\overline{\boldsymbol{A}}^{\alpha}(\boldsymbol{x}, t)= & \left\langle\boldsymbol{A}^{\alpha}(\boldsymbol{x}, t)\right\rangle \equiv \frac{1}{\Delta t} \int_{0}^{\Delta t} \boldsymbol{A}^{\alpha}(\boldsymbol{x}, t+\tau) d \tau \\
= & \frac{1}{\Delta t} \int_{0}^{\Delta t} \sum_{k=1}^{n} \boldsymbol{a}\{\boldsymbol{r}(t+\tau), \boldsymbol{p}(t+\tau)\} \\
& \times \delta\left(\boldsymbol{R}^{k}-\boldsymbol{x}\right) \tilde{\delta}\left(\Delta \boldsymbol{r}^{k \alpha}-\boldsymbol{y}^{\alpha}\right) d \tau .
\end{aligned}
$$

With the help of Eq. (5), the time evolution of the local density function can be obtained as

$$
\begin{aligned}
& \left.\frac{\partial \boldsymbol{A}^{\alpha}}{\partial t}\right|_{\boldsymbol{x}, \boldsymbol{y}^{\alpha}} \\
& =\sum_{k=1}^{n} \frac{d \boldsymbol{a}}{d t} \delta\left(\boldsymbol{R}^{k}-\boldsymbol{x}\right) \tilde{\delta}\left(\Delta \boldsymbol{r}^{k \alpha}-\boldsymbol{y}^{\alpha}\right) \\
& \quad-\nabla_{\boldsymbol{x}} \cdot\left[\sum_{k=1}^{n} \boldsymbol{V}^{k} \otimes \boldsymbol{a} \delta\left(\boldsymbol{R}^{k}-\boldsymbol{x}\right) \tilde{\delta}\left(\Delta \boldsymbol{r}^{k \alpha}-\boldsymbol{y}^{\alpha}\right)\right] \\
& \quad-\nabla_{\boldsymbol{y}^{\alpha}} \cdot\left[\sum_{k=1}^{n} \Delta \boldsymbol{v}^{k \alpha} \otimes \boldsymbol{a} \delta\left(\boldsymbol{R}^{k}-\boldsymbol{x}\right) \tilde{\delta}\left(\Delta \boldsymbol{r}^{k \alpha}-\boldsymbol{y}^{\alpha}\right)\right] .
\end{aligned}
$$

When $A^{\alpha}$ is a conserved property, it results in a local conservation law that governs the time evolution of $A^{\alpha}$. The mathematical representation of conservation equations for mass, linear momentum and energy at atomic scale has been analytically obtained in terms of averaged field quantities
$[23,25,30]$, which are

$$
\begin{aligned}
& \frac{d \bar{\rho}^{\alpha}}{d t}+\bar{\rho}^{\alpha} \nabla_{\mathbf{x}} \cdot \overline{\mathbf{v}}+\bar{\rho}^{\alpha} \nabla_{\mathbf{y}^{\alpha}} \cdot \Delta \overline{\mathbf{v}}^{\alpha}=0 \\
& \bar{\rho}^{\alpha} \frac{d}{d t}\left(\overline{\mathbf{v}}+\Delta \overline{\mathbf{v}}^{\alpha}\right)=\nabla_{\mathbf{x}} \cdot \overline{\boldsymbol{t}}^{\alpha}+\nabla_{\mathbf{y}^{\alpha}} \cdot \overline{\boldsymbol{\tau}}^{\alpha}+\bar{\varphi}^{\alpha} \\
& \bar{\rho}^{\alpha} \frac{d \bar{e}^{\alpha}}{d t}+\nabla_{\mathbf{x}} \cdot\left(-\overline{\boldsymbol{q}}^{\alpha}\right)+\nabla_{\mathbf{y}^{\alpha}} \cdot\left(-\overline{\boldsymbol{j}}^{\alpha}\right) \\
& =\overline{\boldsymbol{t}}^{\alpha}: \nabla_{\mathbf{x}}\left(\overline{\mathbf{v}}+\Delta \overline{\mathbf{v}}^{\alpha}\right)+\overline{\boldsymbol{\tau}}^{\alpha}: \nabla_{\mathbf{y}^{\alpha}}\left(\overline{\mathbf{v}}+\Delta \overline{\mathbf{v}}^{\alpha}\right)+\bar{h}^{\alpha}
\end{aligned}
$$

where the time-interval averaged mass density $\bar{\rho}^{\alpha}$, linear momentum $\bar{\rho}^{\alpha}\left(\bar{v}+\Delta \overline{\boldsymbol{v}}^{\alpha}\right)$, homogeneous atomic stresses $\overline{\boldsymbol{t}}_{(\mathrm{kin})}^{\alpha}+\overline{\boldsymbol{t}}_{(\mathrm{pot})}^{\alpha}$ and inhomogeneous atomic stresses $\overline{\boldsymbol{\tau}}_{(\mathrm{kin})}^{\alpha}+$ $\overline{\boldsymbol{\tau}}_{\text {(pot) }}^{\alpha}$, body force density $\bar{\varphi}^{\alpha}$, internal energy density $\bar{\rho}^{\alpha} \bar{e}^{\alpha}$, the homogeneous part $\overline{\boldsymbol{q}}^{\alpha}$ and inhomogeneous part $\overline{\boldsymbol{j}}^{\alpha}$ of heat flux, and heat source $\bar{h}^{\alpha}$ are defined as

$$
\begin{gathered}
\bar{\rho}^{\alpha}(\boldsymbol{x}, t) \equiv\left\langle\sum_{k=1}^{n} m^{\alpha} \delta\left(\boldsymbol{R}^{k}-\boldsymbol{x}\right) \tilde{\delta}\left(\Delta \boldsymbol{r}^{k \alpha}-\boldsymbol{y}^{\alpha}\right)\right\rangle, \\
\bar{\rho}^{\alpha}\left(\overline{\boldsymbol{v}}+\Delta \overline{\boldsymbol{v}}^{\alpha}\right) \equiv\left\langle\sum_{k=1}^{n} m^{\alpha}\left(\boldsymbol{V}^{k}+\Delta \boldsymbol{v}^{k \alpha}\right) \delta\left(\boldsymbol{R}^{k}-\boldsymbol{x}\right)\right. \\
\left.\times \tilde{\delta}\left(\Delta \boldsymbol{r}^{k \alpha}-\boldsymbol{y}^{\alpha}\right)\right\rangle, \\
\overline{\boldsymbol{t}}_{(\mathrm{kin})}^{\alpha} \equiv-\left\langle\sum_{k=1}^{n} m^{\alpha} \tilde{\boldsymbol{V}}^{k} \otimes \tilde{\boldsymbol{V}}^{k \alpha} \delta\left(\boldsymbol{R}^{k}-\boldsymbol{x}\right)\right. \\
\left.\times \tilde{\delta}\left(\Delta \boldsymbol{r}^{k \alpha}-\boldsymbol{y}^{\alpha}\right)\right\rangle,
\end{gathered}
$$

$$
\overline{\boldsymbol{\tau}}_{(\mathrm{kin})}^{\alpha} \equiv-\left\langle\sum_{k=1}^{n} m^{\alpha} \Delta \tilde{\boldsymbol{v}}^{k \alpha} \otimes \tilde{\boldsymbol{V}}^{k \alpha} \delta\left(\boldsymbol{R}^{k}-\boldsymbol{x}\right)\right.
$$

$$
\left.\times \tilde{\delta}\left(\Delta \boldsymbol{r}^{k \alpha}-\boldsymbol{y}^{\alpha}\right)\right\rangle,
$$

$$
\overline{\boldsymbol{t}}_{(\mathrm{pot})}^{\alpha} \equiv-\frac{1}{2}\left\langle\sum_{k, l=1}^{n} \sum_{\xi, \eta=1}^{v}\left(\boldsymbol{R}^{k}-\boldsymbol{R}^{l}\right)\right.
$$

$$
\left.\otimes \boldsymbol{F}^{k \xi} B\left(k, \xi, l, \eta, \boldsymbol{x}, \boldsymbol{y}^{\alpha}\right)\right),
$$

$$
\begin{gathered}
\overline{\boldsymbol{\tau}}_{(\mathrm{pot})}^{\alpha} \equiv-\frac{1}{2}\left\langle\sum_{k, l=1}^{n} \sum_{\xi, \eta=1}^{v}\left(\Delta \boldsymbol{r}^{k \xi}-\Delta \boldsymbol{r}^{l \eta}\right)\right. \\
\left.\otimes \boldsymbol{F}^{k \xi} B\left(k, \xi, l, \eta, \boldsymbol{x}, \boldsymbol{y}^{\alpha}\right)\right\rangle, \\
\overline{\boldsymbol{\varphi}}^{\alpha} \equiv\left\langle\sum_{k=1}^{n} \boldsymbol{\varphi}^{k \alpha} \delta\left(\boldsymbol{R}^{k}-\boldsymbol{x}\right) \times \tilde{\delta}\left(\Delta \boldsymbol{r}^{k \alpha}-\boldsymbol{y}^{\alpha}\right)\right\rangle,
\end{gathered}
$$




$$
\begin{aligned}
& \bar{\rho}^{\alpha} \bar{e}^{\alpha} \equiv\left\langle\sum_{k=1}^{N_{l}} \sum_{\xi=1}^{N_{a}}\left[\frac{1}{2} m^{\xi}\left(\tilde{\boldsymbol{V}}^{k \xi}\right)^{2}+U^{k \xi}\right] \delta\left(\boldsymbol{R}^{k}-\boldsymbol{x}\right)\right. \\
& \left.\times \tilde{\delta}\left(\Delta \boldsymbol{r}^{k \xi}-\boldsymbol{y}^{\alpha}\right)\right\rangle, \\
& \overline{\boldsymbol{q}}_{\mathrm{kin}}^{\alpha} \equiv-\left\langle\sum_{k=1}^{N_{l}} \sum_{\xi=1}^{N_{a}} \tilde{\boldsymbol{V}}^{k}\left(\frac{1}{2} m^{\xi}\left(\tilde{\boldsymbol{V}}^{k \xi}\right)^{2}+U^{k \xi}\right)\right. \\
& \left.\times \delta\left(\boldsymbol{R}^{k}-\boldsymbol{x}\right) \tilde{\delta}\left(\Delta \boldsymbol{r}^{k \xi}-\boldsymbol{y}^{\alpha}\right)\right\rangle, \\
& \overline{\boldsymbol{q}}_{\mathrm{pot}}^{\alpha} \equiv-\left\langle\frac{1}{2} \sum_{k, l=1}^{N_{l}} \sum_{\xi, \eta=1}^{N_{a}}\left(\boldsymbol{R}^{k}-\boldsymbol{R}^{l}\right) \tilde{\boldsymbol{V}}^{k \xi}\right. \\
& \left.\cdot \boldsymbol{F}^{k \xi} B\left(k, \xi, l, \eta, \boldsymbol{x}, y^{\alpha}\right)\right\rangle, \\
& \overline{\boldsymbol{j}}_{\mathrm{kin}}^{\alpha} \equiv-\left\langle\sum_{k=1}^{N_{l}} \sum_{\xi=1}^{N_{a}} \Delta \tilde{\mathbf{v}}^{\mathrm{k} \xi}\left(\frac{1}{2} m^{\xi}\left(\tilde{\boldsymbol{V}}^{k \xi}\right)^{2}+U^{k \xi}\right)\right. \\
& \left.\times \delta\left(\boldsymbol{R}^{k}-\boldsymbol{x}\right) \tilde{\delta}\left(\Delta \boldsymbol{r}^{k \xi}-\boldsymbol{y}^{\alpha}\right)\right\rangle, \\
& \overline{\boldsymbol{j}}_{\text {pot }}^{\alpha} \equiv-\left\langle\frac{1}{2} \sum_{k, l=1}^{N_{l}} \sum_{\xi, \eta=1}^{N_{a}}\left(\Delta \boldsymbol{r}^{k \xi}-\Delta \boldsymbol{r}^{l \eta}\right) \tilde{\boldsymbol{V}}^{k \xi}\right. \\
& \left.\boldsymbol{F}^{k \xi} B\left(k, \xi, l, \eta, \boldsymbol{x}, y^{\alpha}\right)\right\rangle, \\
& \bar{h}^{\alpha} \equiv\left\langle\sum_{k=1}^{N_{l}} \sum_{\xi=1}^{N_{a}} \tilde{\boldsymbol{V}}^{k \xi} \cdot \varphi^{k \xi} \delta\left(\boldsymbol{R}^{k}-\boldsymbol{x}\right)\right. \\
& \left.\times \tilde{\delta}\left(\Delta \boldsymbol{r}^{k \xi}-\boldsymbol{y}^{\alpha}\right)\right\rangle,
\end{aligned}
$$

where $\boldsymbol{F}^{k \xi}$ is the interatomic force acting on the $k \xi$ atom; $\varphi^{k \alpha}$ is the body force acting the $k \alpha$ atom; $U^{k \xi}$ is the potential energy of the $k \xi$ atom;

$$
\begin{aligned}
\tilde{\boldsymbol{V}}^{k \alpha} \equiv \boldsymbol{V}^{k \alpha}-\overline{\mathbf{v}}-\Delta \overline{\mathbf{v}}^{\alpha}, \quad \tilde{\boldsymbol{V}}^{k} \equiv \boldsymbol{V}^{k}-\overline{\mathbf{v}}, \\
\Delta \tilde{\mathbf{v}}^{k \alpha} \equiv \Delta \mathbf{v}^{k \alpha}-\Delta \overline{\mathbf{v}}^{\alpha}, \\
B\left(k, \xi, l, \eta, \boldsymbol{x}, \boldsymbol{y}^{\alpha}\right) \equiv \int_{0}^{1} d \lambda \delta\left(\boldsymbol{R}^{k} \lambda+\boldsymbol{R}^{l}(1-\lambda)-\boldsymbol{x}\right) \\
\times \tilde{\delta}\left(\Delta \boldsymbol{r}^{k \xi} \lambda+\Delta \boldsymbol{r}^{l \eta}(1-\lambda)-\boldsymbol{y}^{\alpha}\right) .
\end{aligned}
$$

where $\overline{\mathbf{v}}$ is the time interval averaged velocity of the centroid of a unit cell and $\Delta \overline{\mathbf{v}}^{\alpha}$ is the time interval averaged velocity of the $\alpha$ th atom relative to the centroid of the unit cell.
It is worthwhile to note that, with the atomistic definitions of interatomic force and the potential parts of the atomic stresses, one has

$\nabla_{\boldsymbol{x}} \cdot \overline{\boldsymbol{t}}_{(\mathrm{pot})}^{\alpha}+\nabla_{y^{\alpha}} \cdot \overline{\boldsymbol{\tau}}_{(\mathrm{pot})}^{\alpha}=\overline{\boldsymbol{f}}^{\alpha}$,

where $\overline{\boldsymbol{f}}^{\alpha}$ is the interatomic force density acting on the $\alpha$ th atom in the unit cell located at $x$.

\section{Finite element formulation}

In the finite element formulation, we work with time-interval averaged quantities. From now on, for simplicity, we adopt the following abbreviations:

$\ddot{\boldsymbol{u}}^{\alpha} \equiv \frac{d\left(\overline{\boldsymbol{v}}+\Delta \overline{\boldsymbol{v}}^{\alpha}\right)}{d t}, \quad \overline{\boldsymbol{t}}_{(\mathrm{kin})}^{\alpha} \equiv \boldsymbol{t}^{\alpha}, \quad \overline{\boldsymbol{\tau}}_{(\mathrm{kin})}^{\alpha} \equiv \boldsymbol{\tau}^{\alpha}$,

$\overline{\boldsymbol{f}}^{\alpha} \equiv \boldsymbol{f}^{\alpha} / V, \quad \overline{\boldsymbol{\varphi}}^{\alpha} \equiv \boldsymbol{\varphi}^{\alpha} / V$,

where $\boldsymbol{u}^{\alpha}$ is the displacement vector of the $\alpha$ th atom; $V$ is the volume of unit cell. The governing equation, Eq. (9), can now be rewritten as

$m^{\alpha} \ddot{\boldsymbol{u}}^{\alpha}=\left\{\nabla_{\boldsymbol{x}} \cdot \boldsymbol{t}^{\alpha}+\nabla_{\boldsymbol{y}^{\alpha}} \cdot \boldsymbol{\tau}^{\alpha}\right\} V+\boldsymbol{f}^{\alpha}+\boldsymbol{\varphi}^{\alpha}$.

For single-element atomic system, Cheung and Yip [34] and Haile [35] gave the definitions for kinetic stresses $t_{i j}$ and temperature $T$. In consistent with their definitions, for multielement atomic system, we have

$t_{i j}^{\alpha}=-\left\langle m^{\alpha} \tilde{v}_{i}\left(\tilde{v}_{j}+\Delta \tilde{v}_{j}^{\alpha}\right)\right\rangle / V$,

$\tau_{i j}^{\alpha}=-\left\langle m^{\alpha} \Delta \tilde{v}_{i}^{\alpha}\left(\tilde{v}_{j}+\Delta \tilde{v}_{j}^{\alpha}\right)\right\rangle / V$,

$3 k_{B} T^{\alpha}=\left\langle m^{\alpha}\left(\tilde{v}_{i}+\Delta \tilde{v}_{i}^{\alpha}\right)\left(\tilde{v}_{i}+\Delta \tilde{v}_{i}^{\alpha}\right)\right\rangle$.

At high temperature and within harmonic approximation, all modes have the same energy [43]. Following this thinking, we take the assumption, made by Xiong et al. [27], which relates the energies of acoustic modes and optic modes and we further assume that the temperature within a unit cell is uniform. This implies $T^{\alpha}=T(\boldsymbol{x}, t)$ and $\nabla_{y^{\alpha}} \cdot \boldsymbol{\tau}^{\alpha}=0$. Now the governing equation, Eq. (28), can be rewritten as

$m^{\alpha} \ddot{\boldsymbol{u}}^{\alpha}=V \nabla \cdot \boldsymbol{t}^{\alpha}+\boldsymbol{f}^{\alpha}+\boldsymbol{\varphi}^{\alpha}$,

with $t_{i j}^{\alpha}=-\gamma^{\alpha} k_{B} T \delta_{i j} / V, \tau_{i j}^{\alpha}=-\left(1-\gamma^{\alpha}\right) k_{B} T \delta_{i j} / V$ and $\gamma^{\alpha} \equiv m^{\alpha} / \sum_{\alpha=1}^{\nu} m^{\alpha}$. In this work, we are only concerned with 'one-way coupling' with temperature and electromagnetic fields, i.e., the temperature and electromagnetic fields are given as functions of space and time. Then the relevant governing equations are just the balance law for linear momentum:

$m^{\alpha} \ddot{\boldsymbol{u}}^{\alpha}=-\gamma^{\alpha} k_{B} \nabla T+\boldsymbol{f}^{\alpha}+\boldsymbol{\varphi}^{\alpha}$.

In case of temperature being constant, including $T=0$, we can drop the first term in the right hand side of Eq. (33), and 
the governing equation for any $\alpha$ th atom in the $k$ th unit cell can be rewritten as

$m^{\alpha} \ddot{\boldsymbol{u}}(k, \alpha)=\boldsymbol{f}(k, \alpha)+\boldsymbol{\varphi}(k, \alpha)$,

where $\boldsymbol{f}(k, \alpha)$ is all the interatomic force acting on the $\alpha$ th atom in the $k$ th unit cell; $\varphi(k, \alpha)$ is all the body force due to external field acting on the $\alpha$ th atom in the $k$ th unit cell. The effect of non zero temperature should be reflected in the boundary conditions. However, in this work we consider zero-temperature. Therefore, for a system with pair potential, one may rewrite Eq. (34) as

$m^{\alpha} \ddot{\boldsymbol{u}}(k, \alpha)=\sum_{l=1}^{n} \sum_{\beta=1}^{v} \boldsymbol{f}(k, \alpha ; l, \beta)+\boldsymbol{\varphi}(k, \alpha)$,

where $f(k, \alpha ; l, \beta)$ is the interatomic force acting on the $\alpha$ th atom of the $k$ th unit cell due to the interaction with the $\beta$ th atom of the $l$ th unit cell, with the understanding $(k, \alpha) \neq$ $(l, \beta)$. The inner product of Eq. (35) with virtual displacement $\delta \boldsymbol{u}(k, \alpha)$ leads to

$$
\begin{aligned}
& m^{\alpha} \ddot{\boldsymbol{u}}(k, \alpha) \cdot \delta \boldsymbol{u}(k, \alpha) \\
& \quad=\sum_{l=1}^{n} \sum_{\beta=1}^{v} \boldsymbol{f}(k, \alpha ; l, \beta) \cdot \delta \boldsymbol{u}(k, \alpha)+\boldsymbol{\varphi}(k, \alpha) \cdot \delta \boldsymbol{u}(k, \alpha) .
\end{aligned}
$$

Sum over all $\alpha$ and $k$, we obtain the following variational equation, the so-called weak form, as

$$
\begin{aligned}
& \sum_{k=1}^{n} \sum_{\alpha=1}^{v} m^{\alpha} \ddot{\boldsymbol{u}}(k, \alpha) \cdot \delta \boldsymbol{u}(k, \alpha) \\
& \quad=\sum_{k=1}^{n} \sum_{\alpha=1}^{v}\left\{\sum_{l=1}^{n} \sum_{\beta=1}^{v} \boldsymbol{f}(k, \alpha ; l, \beta)+\boldsymbol{\varphi}(k, \alpha)\right\} \cdot \delta \boldsymbol{u}(k, \alpha) .
\end{aligned}
$$

Notice that Eq. (37) can also be expressed as

$$
\begin{aligned}
& \sum_{k=1}^{n} \sum_{\alpha=1}^{v} m^{\alpha} \ddot{\boldsymbol{u}}(k, \alpha) \cdot \delta \boldsymbol{u}(k, \alpha) \\
& =\sum_{k=1}^{n} \sum_{\alpha=1}^{v} \sum_{l=1}^{n} \sum_{\beta=1}^{v} \boldsymbol{f}(l, \beta ; k, \alpha) \cdot \delta \boldsymbol{u}(l, \beta) \\
& \quad+\sum_{k=1}^{n} \sum_{\alpha=1}^{v} \boldsymbol{\varphi}(k, \alpha) \cdot \delta \boldsymbol{u}(k, \alpha) .
\end{aligned}
$$

It is noticed that

$$
\boldsymbol{f}(k, \alpha ; l, \beta)=-\boldsymbol{f}(l, \beta ; k, \alpha),
$$

Therefore Eq. (38) can be rewritten as

$$
\begin{aligned}
& \sum_{k=1}^{n} \sum_{\alpha=1}^{v} m^{\alpha} \ddot{\boldsymbol{u}}(k, \alpha) \cdot \delta \boldsymbol{u}(k, \alpha) \\
& =\frac{1}{2} \sum_{k=1}^{n} \sum_{\alpha=1}^{v} \sum_{l=1}^{n} \sum_{\beta=1}^{v} \boldsymbol{f}(k, \alpha ; l, \beta) \cdot[\delta \boldsymbol{u}(k, \alpha)-\delta \boldsymbol{u}(l, \beta)] \\
& \quad+\sum_{k=1}^{n} \sum_{\alpha=1}^{v} \boldsymbol{\varphi}(k, \alpha) \cdot \delta \boldsymbol{u}(k, \alpha) .
\end{aligned}
$$

Suppose the whole specimen is divided into $N_{e}$ finite elements (8-node solid elements) with $N_{p}$ finite elements nodes, and we approximate Eq. (40) as [30]

$$
\begin{aligned}
& \sum_{I_{p}=1}^{N_{p}} \sum_{\alpha=1}^{v} \frac{V\left(I_{p}\right)}{V}\left\{m^{\alpha} \ddot{\boldsymbol{u}}\left(I_{p}, \alpha\right) \cdot \delta \boldsymbol{u}\left(I_{p}, \alpha\right)-\boldsymbol{\varphi}\left(I_{p}, \alpha\right) \cdot \delta \boldsymbol{u}\left(I_{p}, \alpha\right)\right. \\
& \left.\quad-\frac{1}{2} \sum_{l=1}^{n} \sum_{\beta=1}^{v} f\left(I_{p}, \alpha ; l, \beta\right) \cdot\left[\delta \boldsymbol{u}\left(I_{p}, \alpha\right)-\delta \boldsymbol{u}(l, \beta)\right]\right\}=0,
\end{aligned}
$$

where $V\left(I_{p}\right)$ is the volume associated with the $I_{p}$ th node and $V$ is the volume of a unit cell; $f\left(I_{p}, \alpha ; l, \beta\right)$ is the force acting on the $\alpha$ th atom of the $I_{p}$ th finite element node due to the interaction with the $\beta$ th atom of the $l$ th unit cell. For the purpose of illustration, a 2D schematic picture shows the computational model with finite elements in Fig. 2, in which the large shaded area (volume) $V(J)$ around the $J$ th node means the area (volume) associated with the $J$ th node and the small shaded area (volume) $V$ around the $k$ th unit cell means the area (volume) associated with the $k$ th unit cell.

Through the shape functions, one may have

$\delta \boldsymbol{u}(l, \beta)=\sum_{\lambda=1}^{8} N^{\lambda}(l) \delta \boldsymbol{U}^{\beta}(l, \lambda)$,

where $N^{\lambda}(l)$ is the $\lambda$ th shape function evaluated at the center of the $l$ th unit cell; $\delta \boldsymbol{U}^{\beta}(l, \lambda)$ is the virtual displacement of the $\beta$ th atom of $\lambda$ th node of the element where the $l$ th unit cell is located.

Now one may rewrite Eq. (41) as

$$
\begin{gathered}
\sum_{I_{p}=1}^{N_{p}} \sum_{\alpha=1}^{v} \frac{V\left(I_{p}\right)}{V}\left\{m^{\alpha} \ddot{\boldsymbol{u}}\left(I_{p}, \alpha\right) \cdot \delta \boldsymbol{u}\left(I_{p}, \alpha\right)-\boldsymbol{\varphi}\left(I_{p}, \alpha\right)\right. \\
\cdot \delta \boldsymbol{u}\left(I_{p}, \alpha\right)-\frac{1}{2} \sum_{l=1}^{n} \sum_{\beta=1}^{v} \boldsymbol{f}\left(I_{p}, \alpha ; l, \beta\right) \\
\left.\cdot\left[\delta \boldsymbol{u}\left(I_{p}, \alpha\right)-\sum_{\lambda=1}^{8} N^{\lambda}(l) \delta \boldsymbol{U}^{\beta}(l, \lambda)\right]\right\}=0 .
\end{gathered}
$$

Let

$J_{p}(l, \lambda) \equiv I J K(\lambda, l)$, 
Fig. 2 Computational model with finite elements and unit cells

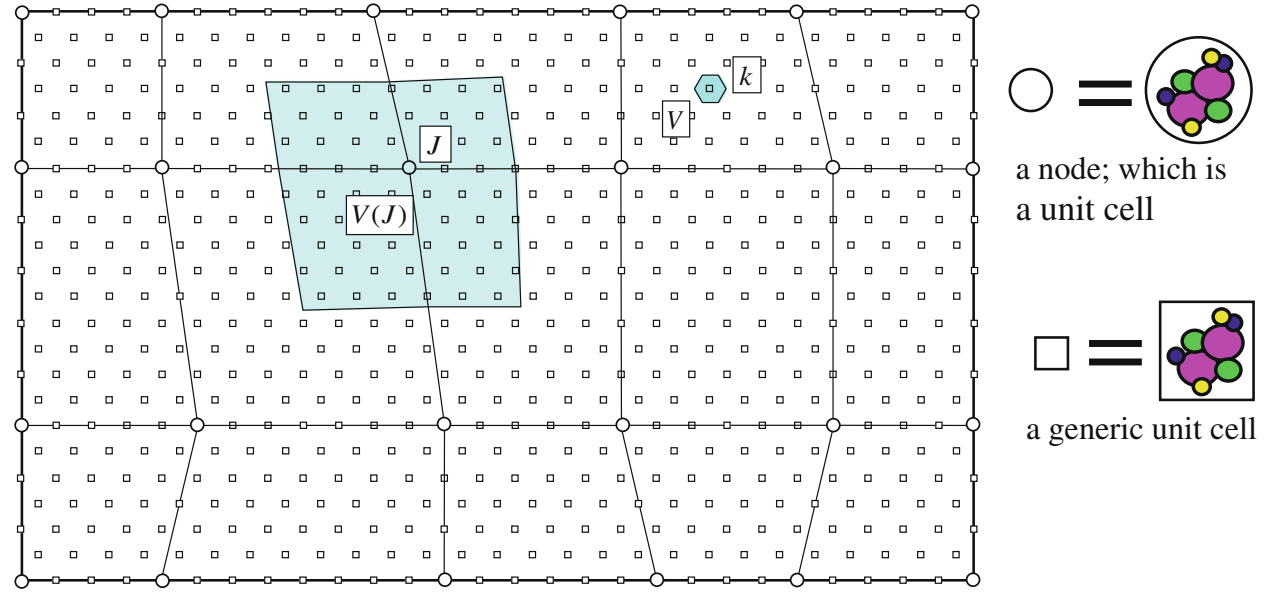

where $I J K$ is the connectivity of the finite element model, i.e., $\operatorname{IJK}(\lambda, l)$ is the global nodal number of the $\lambda$ th node of the element that the $l$ th unit cell is located. Equation (43) can be rewritten as

$$
\begin{aligned}
& \sum_{I_{p}=1}^{N_{p}} \sum_{\alpha=1}^{v} \frac{V\left(I_{p}\right)}{V}\left\{\left[m^{\alpha} \ddot{\boldsymbol{u}}\left(I_{p}, \alpha\right)-\boldsymbol{\varphi}\left(I_{p}, \alpha\right)\right] \cdot \delta \boldsymbol{U}^{I_{p}, \alpha}\right. \\
& -\frac{1}{2} \sum_{l=1}^{n} \sum_{\beta=1}^{v} \boldsymbol{f}\left(I_{p}, \alpha ; l, \beta\right) \\
& \left.\cdot\left[\delta \boldsymbol{U}^{I_{p}, \alpha}-\sum_{\lambda=1}^{8} N^{\lambda}(l) \delta \boldsymbol{U}^{J_{p}(l, \lambda), \beta}\right]\right\}=0 .
\end{aligned}
$$

We request that Eq. (45) remains valid for any arbitrary virtual displacement, $\delta U_{i}^{I_{p}, \alpha}$. Properly going through the process of nodal force assembly, Eq. (45) can be expressed as

$$
\begin{aligned}
& m^{\alpha} \ddot{\boldsymbol{u}}\left(I_{p}, \alpha\right)=\boldsymbol{F}\left(I_{p}, \alpha\right)+\boldsymbol{\varphi}\left(I_{p}, \alpha\right) \\
& \quad\left(I_{p}=1,2,3, \ldots, N_{p} ; \alpha=1,2,3, \ldots, v\right),
\end{aligned}
$$

where $\boldsymbol{F}\left(I_{p}, \alpha\right)$ and $\boldsymbol{\varphi}\left(I_{p}, \alpha\right)$ are the interatomic force and the body force acting on $\alpha$ th atom of the $I_{p}$ th node, respectively. It is noticed that the mass matrix of Eq. (46) is a diagonal matrix.

Bases on the continuum field theory, a computer software, named CMM, has been developed. The CMM is capable to solve nonlinear, nonlocal, large strain and dynamic problems. It was based on Gauss-point-integration. The integration method developed in this work may be referred to as the direct nodal point integration method, or collocation method. Comparing with Gauss-point-integration method, collocation method simplifies the calculation of atomic forces and dramatically reduces the computational effort. Another advantage of the direct nodal point integration is that when the element size is reduced to the size of a unit cell, the program can solve problems exactly at atomic level as molecular dynamics simulation.

A comparison of classical continuum mechanics, micromorphic theory, the newly formulated field theory and MD are summarized in Table 1. As is shown in Fig. 1, the atomic view of a crystal is as a periodic arrangement of local atomic bonding units. For multi-element systems, the space lattice is macroscopically homogeneous, whereas embedded in each lattice point is a group of discrete atoms, the smallest structural unit of the crystal. Classic continuum mechanics views a crystal as a homogeneous and continuous medium, in which the basic structural unit of the crystal is taken without structure and is idealized as point mass, and the internal deformation is ignored. Its application is thus limited to homogeneous or macroscopic problems. Eringen and Suhubi [36] established the well-known Micromorphic theory in which the material is envisioned as a continuous collection of deformable particles; each particle has finite size and 9 internal degrees of freedom describing the internal deformation of the particle. Compared with classical continuum mechanics, Micromorphic theory extends the application region of continuum theory to microscopic time and length scales. However, the assumption of a continuous structure and deformation of the particles makes it difficult to describe complex crystalline materials. Also it is very difficult to obtain material parameters for complex crystals in micromorphic theory, the current authors proposed an optimization algorithm to obtain materials constants for single crystal silicon and diamond [37].

The newly formulated theory [23-31] views a crystalline material as a continuous collection of lattice points, while embedded with each lattice point is a group of discrete atoms. Hence this further expands the application region of micromorphic theory to the atomic scale. While it retains most of the features of an atomic many-body dynamics, the new field theory is able to scale up in modeling and simulations 
Table 1 Comparison of classical continuum theory, micromorphic, the new field theory, and MD

\begin{tabular}{|c|c|c|c|c|c|}
\hline 20000 & $\begin{array}{c}\text { Atomic } \\
\text { structure } \\
\text { unit }\end{array}$ & $\begin{array}{c}\text { Lattice } \\
\text { deformation }\end{array}$ & $\begin{array}{c}\text { Internal } \\
\text { deformation }\end{array}$ & Applications & Limitations \\
\hline $\begin{array}{c}\text { Classical } \\
\text { Continuum } \\
\text { Theory }\end{array}$ & Mass point & Continuous & None & $\begin{array}{c}\text { Macroscopic } \\
\text { or } \\
\text { homogeneous } \\
\text { problems }\end{array}$ & $\begin{array}{c}\text { For } \\
\text { homogeneous } \\
\text { problems }\end{array}$ \\
\hline $\begin{array}{l}\text { Micromorphic } \\
\text { Theory }\end{array}$ & $\begin{array}{l}\text { A finite size } \\
\text { continuum }\end{array}$ & Continuous & Continuous & $\begin{array}{l}\text { Nano to macro } \\
\text { scale }\end{array}$ & $\begin{array}{l}\text { Difficult for } \\
\text { complex } \\
\text { crystals }\end{array}$ \\
\hline $\begin{array}{c}\text { The New Field } \\
\text { Theory }\end{array}$ & Discrete & Continuous & Discrete & $\begin{array}{c}\text { Nano to macro } \\
\text { scale }\end{array}$ & $\begin{array}{l}\text { Computationa } \\
\text { more costly } \\
\text { than } \\
\text { Micromorphic }\end{array}$ \\
\hline $\begin{array}{c}\text { Molecular } \\
\text { dynamics } \\
\text { (MD) }\end{array}$ & Discrete & Discrete & Discrete & $\begin{array}{l}\text { Nano to micro } \\
\text { scale }\end{array}$ & $\begin{array}{l}\text { Length/time } \\
\text { scale is } \\
\text { limited }\end{array}$ \\
\hline
\end{tabular}

of various physical systems and phenomena. Note that all the quantities in the balance equations are time-interval averaged quantities. This is one major difference between the field representation and MD simulation: MD solves for instantaneous quantities first and then obtains averaged quantities through statistical averaging, while the field theory solves directly for averaged quantities. Another fundamental difference between MD and the field theory is that the lattice deformation is assumed to be continuous with respect to $\mathbf{x}$ in the physical space. This assumption requires that the smallest $\Delta x$, e.g. the mesh size, should be no less than the lattice spacing. In the case that $\Delta x$ equals lattice spacing; the field theory has the same degrees of freedom as MD.

\section{Simulation methodology}

In this work, we adopt the Finnis-Sinclair Model [38] for material bcc Fe. The potential energy of the Finnis-Sinclair Model (FSM) and the Embedded Atom Model (EAM) has the following general form:

$U=\frac{1}{2} \sum_{i=1}^{N} \sum_{j=1}^{N} V_{i j}\left(r_{i j}\right)+\sum_{i=1}^{N} F\left(\rho_{i}\right)$

where $F\left(\rho_{i}\right)$ is a functional describing the energy of embedding, defined as

$\rho_{i}=\sum_{j \neq i}^{N} \rho_{i j}\left(r_{i j}\right), \quad \boldsymbol{r}_{i j} \equiv \boldsymbol{r}_{i}-\boldsymbol{r}_{j}, \quad r_{i j} \equiv\left|\boldsymbol{r}_{i j}\right|$

Finnis and Sinclair used the second-moment approximation of the tight-binding formulation to derive a nonlinear energy function. The Finnis-Sinclair potential is defined as

$V_{i j}=\left(r_{i j}-c\right)^{2}\left(c_{0}+c_{1} r_{i j}+c_{2} r_{i j}^{2}\right)$

$\rho_{i j}\left(r_{i j}\right)=\left(r_{i j}-d\right)^{2}+\beta \frac{\left(r_{i j}-d\right)^{3}}{d}$

$F\left(\rho_{i}\right)=-A \sqrt{\rho_{i}}$,

with parameters $c_{0}, c_{1}, c_{2}, c, A, d, \beta$ from the reference [39]; both $c$ and $d$ are cutoffs.

We rewrite the EAM/FSM potential as:

$U=U_{1}+U_{2}, \quad U_{1}=\frac{1}{2} \sum_{i=1}^{N} \sum_{j=1}^{N} V_{i j}\left(r_{i j}\right)$,

$U_{2}=\sum_{i=1}^{N} F\left(\rho_{i}\right)$

The atomic forces for FSM can be obtained as:

$$
\begin{aligned}
\boldsymbol{F}^{k}=- & \frac{\partial U_{1}}{\partial \boldsymbol{r}_{k}}-\frac{\partial U_{2}}{\partial \boldsymbol{r}_{k}}, \\
-\frac{\partial U_{1}}{\partial \boldsymbol{r}_{k}}= & -\sum_{j=1, j \neq k}^{N}\left\{2\left(r_{k j}-c\right)\left(c_{0}+c_{1} r_{k j}+c_{2} r_{k j}^{2}\right)\right. \\
& \left.+\left(r_{k j}-c\right)^{2}\left(c_{1}+2 c_{2} r_{k j}\right)\right\} \frac{\boldsymbol{r}_{k j}}{r_{k j}} \\
-\frac{\partial U_{2}}{\partial \boldsymbol{r}_{k}}= & \sum_{j=1, j \neq k}^{N} \frac{A}{2}\left(\frac{1}{\sqrt{\rho_{k}}}+\frac{1}{\sqrt{\rho_{j}}}\right) \\
& \times\left\{2\left(r_{k j}-d\right)+3 \beta \frac{\left(r_{k j}-d\right)^{2}}{d}\right\} \frac{\boldsymbol{r}_{k j}}{r_{k j}}
\end{aligned}
$$




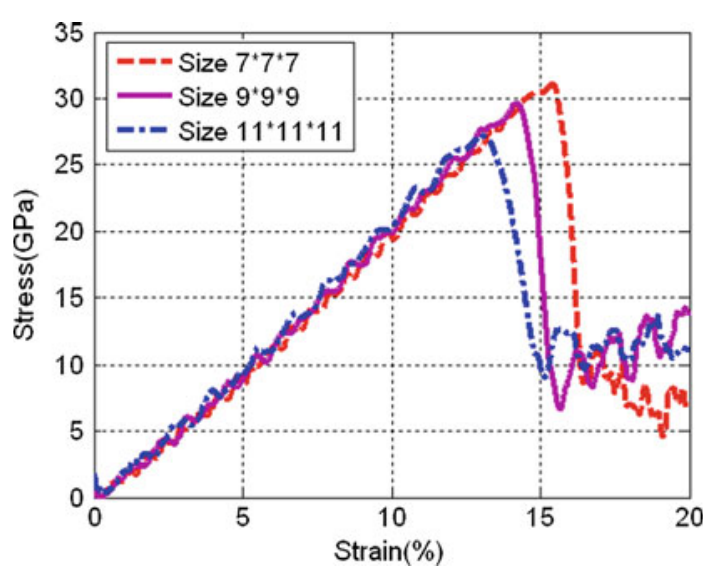

Fig. 3 Stress-strain relations from compressive loading at different sizes

\section{Simulation results}

In this work, we first refine the finite element mesh to atomic scale to investigate the mechanical behavior of bcc Fe and then we scale up the size of the model to simulate wave propagation. To further illustrate the field theory, we create a multiscale model to simulate a dynamic crack propagation process. In these analyses, atomic units are used throughout unless otherwise explicitly specified. The lattice constant of bcc Fe is $a=2.87 \AA$.

\subsection{Atomistic simulation of mechanical behavior of bcc $\mathrm{Fe}$}

As we mentioned earlier, when we refine the finite element size to the size of a unit cell, the field theory is automatically reduced to an atomic theory. In this simulation, the single crystal bcc Fe subjected to compressive loading is simulated by the formulated multiscale field theory. The specimen consists of $7 \times 7 \times 7,9 \times 9 \times 9,11 \times 11 \times 11$ unit cells with two atoms per unit cell. Figure 3 shows the stress-strain relations for different size specimens under compressive loading conditions. As the system size increase from $7 \times 7 \times 7,9 \times 9 \times 9$, to $11 \times 11 \times 11$, the compressive strength decreases from $31,29.5$, to $27.5 \mathrm{GPa}$, the Young's modules values were progressively larger in our case.

Figure 4 shows the structural evolution during the linearly increasing loading stage and the constant loading (holding) stage as in Eqs. (45) and (46).

$$
\begin{aligned}
& u_{z}(x, y, 0, t)= \begin{cases}U^{0} t / T & \text { if } t \leq t^{0} \\
U^{0} & \text { if } t^{0}<t \leq T\end{cases} \\
& u_{z}(x, y, L, t)= \begin{cases}-U^{0} t / T & \text { if } t \leq t^{0} \\
-U^{0} & \text { if } t^{0}<t \leq T\end{cases}
\end{aligned}
$$

where $U^{0}=\varepsilon L / 2$ with $\varepsilon$ is the applied strain, $L$ is the total length of the specimen, $t^{0}=0.6 T$.

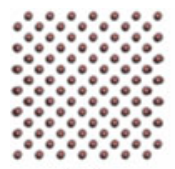

(a)

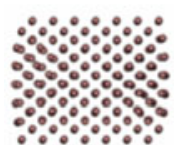

(e)

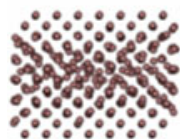

(i)

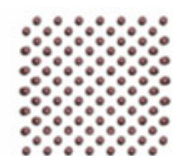

(b)

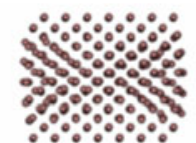

(f)

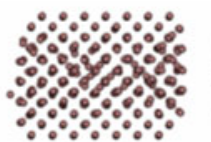

(j)

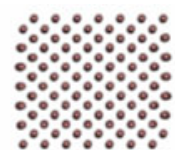

(c)

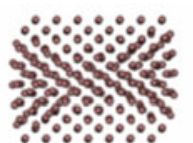

(g)

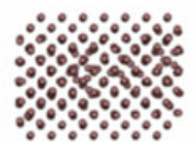

(k)

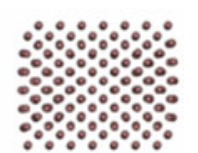

(d)

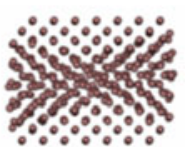

(h)

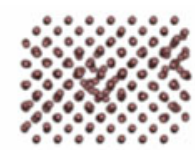

(l)
Fig. 4 Crystal structure evolution during compressive loading: a $t=0$, b $t=2.0 \times 10^{5}, \mathbf{c} t=4.0 \times 10^{5}, \mathbf{d} t=6.0 \times 10^{5}, \mathbf{e} t=6.2 \times 10^{5}$, $\mathbf{f} t=6.3 \times 10^{5}, \mathbf{g} t=6.4 \times 10^{5}, \mathbf{h} t=6.5 \times 10^{5}, \mathbf{i} t=7.0 \times 10^{5}$, $\mathbf{j} t=8.0 \times 10^{5}, \mathbf{k} t=9.0 \times 10^{5}, \mathbf{l} t=10 \times 10^{5}$
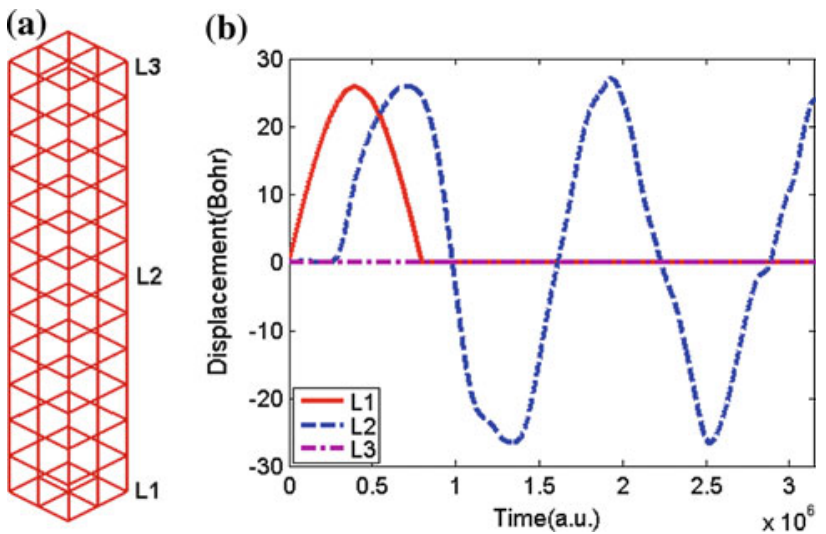

Fig. 5 a FE mesh. b Average displacements at different layers versus time

It is interesting to see, at the holding stage, while the loading remains constant, the deformed structure evolves. As a result of the motion of dislocations, the dislocation emitted from the sides of the crystal. From the simulation results, one can find the plastic deformation has been generated during the loading stage, and the crystal structure becomes ordered again during the steady state holding stage.

\subsection{Wave propagation at continuum level}

After the atomic simulation of bcc Fe system, we then scale up the finite element size to submicron scale to study wave propagation. The finite element model of the specimen for wave propagation has $2 \times 2 \times 12$ elements as shown in Fig. $5 \mathrm{a}$. 8 -node solid elements are used; the total number of unit cell is 405,121 with two atoms per unit cell.

There are totally 80,000 time steps with $\Delta t=40$ atomic units $\left(\tau_{o} \equiv a_{o}^{2} m_{e} \hbar^{-1}\right)$. The displacement specified boundary 
conditions are applied at the two ends of the specimen as

$u_{z}(x, y, 0, t)=\left\{\begin{array}{ll}U^{0} \sin \omega t & \text { if } t \leq t^{0} \\ 0 & \text { if } t^{0}<t \leq T\end{array}\right.$,

$u_{z}(x, y, L, t)=0$,

where $U^{0}=\varepsilon L$ with $\varepsilon=0.02 ; \omega=\pi / t^{o}$ with $t^{0}=T / 4$; the total length of the specimen is $L \approx 69.2 \mathrm{~nm}$; the total simulation time is $T=3,200,000 \tau_{o} \approx 77.5 \mathrm{ps}$. The phenomenon of wave propagation has been observed in the simulation. Figure $5 \mathrm{~b}$ shows the displacements at different layers of the specimen, the top surface of the specimen is fixed, the half-sine wave is generated at the bottom surface and we captured the wave at the central layer of the specimen. Based on the simulation results, the wave speed of the traveling wave in the bcc iron is estimated to be $4770 \mathrm{~m} / \mathrm{s}$, which is in reasonable agreement with the experimental value around $v_{L}=\sqrt{C_{11} / \rho}=5326 \mathrm{~m} / \mathrm{s}$ [40]. The predicted wave speed is lower than the experimental value. The possible reason is that maybe the potential we are using is not good enough.

\subsection{Multiscale modeling and simulation of dynamic crack propagation}

The traditional continuum-based theories of fracture mechanics provide the basic computational and modeling tools for studying the fracture processes. These theories provide a variety of energy and stress criteria for computing the conditions for further growth of a static crack on the verge of extension. Despite their valuable contributions, a modeling of a fracture process based exclusively on these theories is not, however, capable of accounting for all of the experimentally observed characteristics of the crack dynamics or the crack-surface topography. These microscopic properties of materials are often determined by events on the atomic scale. So, for the detailed understanding of fracture and crack propagation, we require an understanding in the atomic scale. But atomistic studies of fracture are computationally rather demanding. Again, in the region away from the crack-tip we do not need the atomic scale discretization. This leads to the use of a multiscale modeling approach that couples the crack propagation across several length scales within one unified and seamless model, which would be able to provide deeper insights into the peculiarities of the crack dynamics.

In the simulation, we decompose the specimen into three regions within one unified and seamless model, the top and bottom regions are continuum regions with 100 finite elements each. In the critical region, the finite element is scaled exactly down to atomic level as shown in Fig. 6, there are 900 finite element which includes 1105 unit cells. An edge crack of finite length is initiated at the left central region of the specimen. The crick tip is right in the center of the specimen. Our aim is to set up a scheme to apply a general

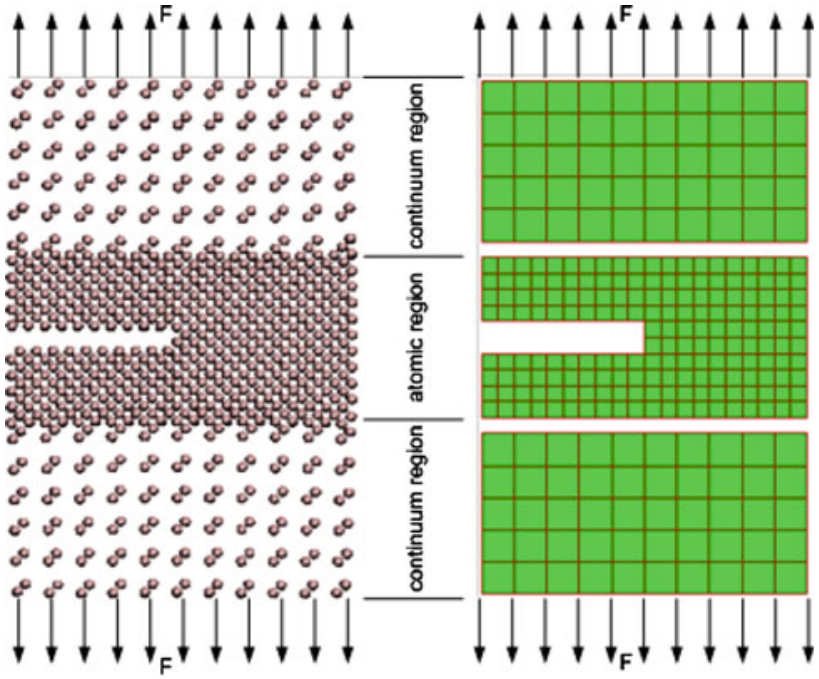

Fig. 6 A multiscale model for crack propagation

external load to a finite-size system. We consider the case in which an external load, ideally applied away from the region of the system where a microcrack is placed. In the case, the force boundary conditions are applied on the top and bottom planes.

Figure 7 shows the dynamic crack propagation process in mode I. It is clearly showed the crack propagated from the central region all the way to the right boundary and finally the specimen is fractured into two parts. Deformation twinning at the crack tip has been observed from the simulation (see Fig. 7e, f), which has been reported in Hai and Tadmor [41], Guo and Zhao [42]. The simulation also proved that, when the element size reduces to the size of a unit cell, the field theory is automatically reduced to an atomic theory. This thus naturally leads to a concurrent atomic/continuum model without the need for scale decoupling or a region of hand-shaking. In the multiscale continuum field theory, both MD simulation and continuum modeling techniques can be utilized.

\section{Discussions and summary}

This paper presents a multiscale continuum field theory and the governing equations for systems with given temperature field. Numerical simulations based on the field theory are performed to investigate the mechanical behaviors of bcc Fe. The phenomenon of wave propagation has been simulated. With the multiscale continuum field theory, we have simulated the crack propagation in a multiscale model.

The QC method has been used to study a variety of fundamental aspects of deformation in crystalline solids at zero temperature based on Cauchy-Born elasticity. In most works based on QC method, triangular finite elements or tetrahedral 
Fig. 7 Dynamic crack propagation process in multiscale model (mode I):

$\mathbf{a} t=0, \mathbf{b} t=5.0 \times 10^{5}$,

c $t=1.0 \times 10^{6}$,

d $t=1.25 \times 10^{6}$,

e $t=1.5 \times 10^{6}$

f $t=1.625 \times 10^{6}$,

g $t=1.75 \times 10^{6}$,

h $t=1.875 \times 10^{6}$,

i $t=1.95 \times 10^{6}$,

j $t=2.0 \times 10^{6}$,

$\mathbf{k} t=2.05 \times 10^{6}$,

$\mathbf{l} t=2.125 \times 10^{6}$

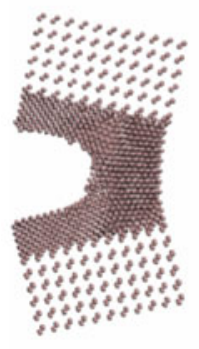

(g)

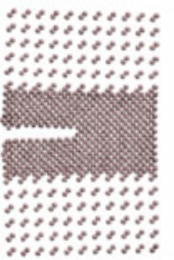

(b)

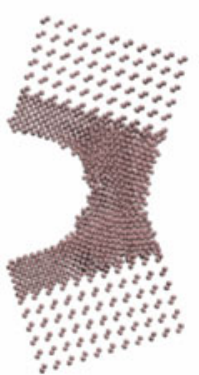

(h)

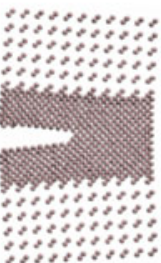

(c)

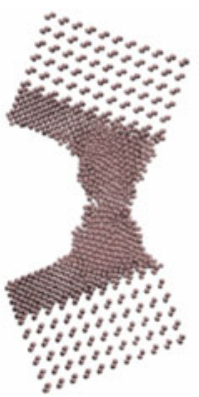

(i)

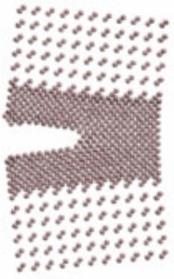

(d)

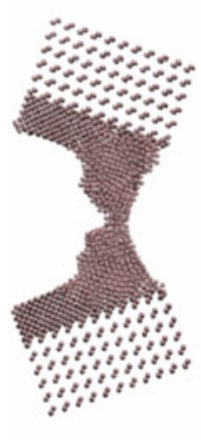

(j)

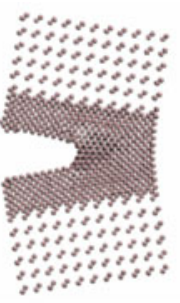

(e)

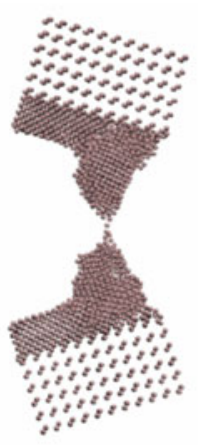

(k)

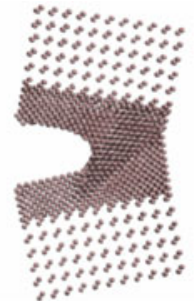

(f) finite elements for continuum region is adopted in $2 \mathrm{D}$ or $3 \mathrm{D}$ simulation, thereby leading to a locally-uniform deformation gradient in the continuum region. Linear interpolation functions in triangular or tetrahedral finite element require only one single Gauss-point for numerical quadrature. As a consequence, the application of the Cauchy-Born rule (CBR) implies that in the energy calculation the summation over the number of lattice sites boils down to the number of finite elements. Therefore, there will be a limitation for QC methods due to the validity of the kinematic assumption of CBR, i.e., to determine the state when a transition to non-affine deformations is possible due to instabilities or inhomogeneous of the underlying atomic system. Comparing with QC, this multiscale continuum field theory has a complete and comprehensive set of balance laws within a single theoretical framework. Secondly, the internal deformation in each unit cell has been naturally built in this field theory, thereby leading to study more complex crystal e.g. PbTiO3, BaTiO3.

One fundamental difference between the continuum field theory and molecular dynamics (MD) simulation is that the governing equations in the field theory are in terms of continuous density functions and the lattice deformation is assumed to be continuous with respect to $\boldsymbol{x}$. Therefore, different meshes can be used in regions of different concerns, and if needed, mesh in critical region can be refined to the atomic scale, which is clearly described in Eqs. (34) and (46). Equation (34) is the basis for atomic simulation, since it is in terms of unit cells, while Eq. (46) is the basis for finite element simulation, since it is in terms of finite element nodes. It has been proven that, when the element size reduces to the size of a unit cell, the field theory is automatically reduced to an atomic theory. This thus naturally leads to a concurrent atomic/continuum model without the need for scale decoupling or a region of hand-shaking. In the multiscale continuum field theory, both MD simulation and continuum modeling techniques can be utilized. As a result, the field theory based simulations are computationally more efficient for statistical, finite size and finite temperature problems, for simultaneously large length/time scale phenomena, and especially, for dynamic, time-dependent and non-equilibrium phenomena and systems. This demonstrates the advantage and applicability of the continuum field theory.

Acknowledgments The authors acknowledge the support by National Science Foundation under Award Number CMMI-0646674.

Open Access This article is distributed under the terms of the Creative Commons Attribution Noncommercial License which permits any noncommercial use, distribution, and reproduction in any medium, provided the original author(s) and source are credited.

\section{References}

1. Stan M, Yip S (2009) Design and evaluation of nuclear fuels and structural materials: predictive modeling and high-performance simulations, white paper for the Joint Office of Science and Office of Nuclear Energy Workshop on Advanced Modeling and Simulation for Nuclear Fission Energy Systems, Washington DC

2. Tadmor EB, Ortiz M, Phillips R (1996) Quasicontinuum analysis of defects in solids. Phil Mag A 73:1529-1563 
3. Ericksen JL (1984) The Cauchy and born hypothesis for crystals. In: Gurtin M (ed) Phase transformations and material instabilities in solids. Academic Press, New York, pp 61-77

4. Rudd RE, Broughton JQ (1998) Coarse-grained molecular dynamics and atomic limit of finite elements. Phys Rev B 58:5893-5896

5. Rudd RE, Broughton JQ (2000) Concurrent coupling of length scales in solid state systems. Phys Stat Sol 217:251-291

6. Abraham F, Broughton J, Bernstein N, Kaxiras E (1998) Spanning the length scales in dynamic simulation. Comput Phys 12:538-546

7. Broughton J, Bernstein N, Kaxiras E, Abraham F (1999) Concurrent coupling of length scales: methodology and application. Phys Rev B 60:2391-2403

8. Rudd RE, Broughton JQ (1999) Atomistic simulation of MEMS resonators trough the coupling of length scales. J Model Simul Microsyst 1(1):29-38

9. Li XT, E W (2005) Multiscale modeling of dynamics of solids at finite temperature. J Mech Phys Solids 53:1650-1685

10. E W, Engquist B (2003) The heterogeneous multi-scale methods. Comm Math Sci 1(1):87-132

11. Wagner GJ, Karpov EG, Liu WK (2004) Molecular dynamics boundary conditions for regular crystal lattices. Comput Methods Appl Mech Eng 193(17-20):1579-1601

12. Qian D, Wagner GJ, Liu WK (2004) A multiscale projection method for the analysis of carbon nanotubes. Comput Methods Appl Mech Eng 193:1603-1632

13. Wagner GJ, Liu WK (2003) Coupling of atomic and continuum simulations using a bridging scale decomposition. J Comput Phys 190:249-274

14. Karpov EG, Yu H, Park H, Liu WK, Wang J, Qian D (2006) Multiscale boundary conditions in crystalline solids: theory and application to nanoindentation. Int J Solids Struct 43(21):6359-6379

15. Park HS, Karpov EG, Liu WK, Klein PA (2005) The bridging scale for three-dimensional atomistic/continuum coupling. Phil Mag 85(1):79-113

16. Xiao SP, Belytschko T (2004) A bridging domain method for coupling continua with molecular dynamics. Comput Methods Appl Mech Eng 193:1645-1669

17. Vernerey FJ, Liu WK, Moran B (2007) Multi-scale micromorphic theory for hierarchical materials. J Mech Phys Solid 55(12):26032651

18. Vernerey FJ, Liu WK, Moran B, Olson GB (2008) A micromorphic model for the multiple scale failure of heterogeneous materials. J Mech Phys Solids 56(4):1320-1347

19. McVeigh C, Liu WK (2008) Linking microstructure and properties through a predictive multiresolution continuum. Comput Methods Appl Mech Eng 197:3268-3290

20. McVeigh C, Liu WK (2009) Multiresolution modeling of ductile reinforced brittle composites. J Mech Phys Solids 57:244-267

21. To AC, Li S (2005) Perfectly matched multiscale simulations. Phys Rev B 72:035414
22. Li S, Liu X, Agrawal A, To AC (2006) Perfectly matched multiscale simulations for discrete systems: extension to multiple dimensions. Phys Rev B 74:045418

23. Chen Y, Lee JD (2005) Atomistic formulation of a multiscale theory for nano/micro physics. Phil Mag 85:4095-4126

24. Chen Y (2006) Local stress and heat flux in atomistic systems involving three-body forces. J Chem Phys 124:054113

25. Chen Y, Lee JD (2006) Conservation laws at nano/micro scales. J Mech Mater Struct 1:681-704

26. Chen Y, Lee JD, Xiong L (2006) Stresses and strains at nano/micro scales. J Mech Mater Struct 1:705-723

27. Xiong L, Chen Y, Lee JD (2007) Atomistic simulation of mechanical properties of diamond and silicon by a field theory. Model Simul Mater Sci Eng 15:535-551

28. Lei Y, Lee JD, Zeng X (2008) Response of a rocksalt crystal to electromagnetic wave modeled by a multiscale field theory. Interact Multiscale Mech 1(4):467-476

29. Chen Y (2009) Reformulation of microscopic balance equations for multiscale materials modeling. J Chem Phys 130(13):134706

30. Lee JD, Wang XQ, Chen Y (2009) Multiscale material modeling and its application to a dynamic crack propagation problem. Theor Appl Fracture Mech 51:33-40

31. Lee JD, Wang XQ, Chen Y (2009) Multiscale computational for nano/micro material. J Eng Mech 135:192-202

32. Irvine JH, Kirkwood JG (1950) The statistical theory of transport processes. IV. The equations of hydrodynamics. J Chem Phys 18:817

33. Hardy RJ (1982) Formulas for determining local properties in molecular-dynamics simulations: shock waves. J Chem Phys 76(1):622-628

34. Cheung KS, Yip S (1991) Atomic-level stress in an inhomogeneous system. J Appl Phys 70(10):5688-5690

35. Haile JM (1992) Molecular dynamics simulation. Wiley, New York

36. Eringen AC, Suhubi ES (1964) Nonlinear theory of simple microelastic solids-I. Int J Eng Sci 2:189-203

37. Zeng XW, Chen YP, Lee JD (2006) Determining material constants in nonlocal micromorphic theory through phonon dispersion relations. Int J Eng Sci 44:1334-1345

38. Finnis MW, Sinclair JE (1984) A simple empirical N-body potential for transition metals. Phil Mag A 50(1):45-55

39. Finnis MW, Sinclair JE (1986) Erratum: a simple empirical N-body potential for transition metals. Phil Mag A 53(1):161

40. Klotz S, Braden M (2000) Phonon dispersion of bcc iron to $10 \mathrm{GPa}$. Phys Rev Lett 85:3209-3212

41. Hai S, Tadmor EB (2003) Deformation twinning at aluminum crack tips. Acta Materialia 51:117-131

42. Guo Y-F, Zhao D-L (2007) Atomistic simulation of structure evolution at crack tip in bcc-iron. Mater Sci Eng A 448:281-286

43. Dove M (1993) Introduction to lattice dynamics. Cambridge University Press, Cambridge 